\section{Potential of Germline CDKN2A I49T as a Targetable Driver Mutation: Prolonged Control of Refractory Osteosarcoma with CDK4/6 Inhibitor in a Familial Cancer}

\author{
Omid S Tehrani ${ }^{1^{*}}$, Haifaa Abdulhaq ${ }^{2}$ and Celia D Delozier ${ }^{2}$ \\ ${ }^{1}$ Division of Oncology, Department of Medicine, Stanford University School of Medicine, Stanford, CA, USA \\ ${ }^{2}$ Division of Oncology, Department of Medicine, UCSF Fresno, CA, USA
}

"Corresponding author: Dr. Omid S Tehrani, Division of Oncology, Department of Medicine, Stanford University School of Medicine, Stanford, CA, USA, Tel: (408)426-4900; E-mail: hematology123@gmail.com

Received date: January 02, 2019; Accepted date: January 22, 2019; Published date: January 25, 2019

Copyright: (02019 Tehrani OS, et al. This is an open-access article distributed under the terms of the Creative Commons Attribution License, which permits unrestricted use, distribution, and reproduction in any medium, provided the original author and source are credited.

\begin{abstract}
Objective: Identifying the potential of germline CDKN2A $149 T$ (also known as p.149T: ATC>ACC) in familial cancer and its potential as a targetable driver mutation in carcinogenesis.

Method: Germline mutational analysis was done using commercially available next generation sequencing (NGS) in kindred affected by cancers of lung, throat, gastrointestinal stromal tumor (GIST) and osteosarcoma using. Treatment of chemo-refractory osteosarcoma was done with CDK4/6 inhibitor palbociclib. Monitoring of response was done by serial computed tomography (CT) imaging.

Results: Two affected members in the kindred, one with GIST and one with osteosarcoma were tested and proven positive for germline CDKN2A 149T alteration. The patient with osteosarcoma experienced progression of the disease despite multiple surgical resections and combination chemotherapy. Patient had a sustainable response to CDK4/6 inhibitor palbociclib, with disease controlled for more than a year.
\end{abstract}

Conclusion: These findings suggested a familial cancer syndrome associated with germline CDKN2A I49T and showed its potential as a targetable driver mutation.

Keywords: Germline mutational analysis; Next generation sequencing; Osteosarcoma

Abbreviations: FAMMM: Familial Atypical Multiple Mole Melanoma; PARP: Poly (ADP-ribose) Polymerase; IHC: ImmuneHisto-Chemistry; GIST: Gastrointestinal Stromal Tumor

\section{Introduction}

$C D K N 2 A$ is frequently altered in a variety of cancers. It occurs as somatic and germline mutations. CDKN2A mutations have been reported in rare osteosarcoma cases [1]. Germline CDKN2A mutations are reported in Familial Atypical Multiple Mole Melanoma (FAMMM). FAMMM patients frequently have multiple hyper pigmented nevi and higher frequency of melanoma and pancreatic cancer [2]. Brain tumors, head/neck and skin cancers have also been reported in mutation carriers [3-5].

At the cellular level, $C D K N 2 A$ gene encodes a variety of proteins including p14 and p16. Clinical significance of CDKN2A I49T, which in turn affects p16 and p14, is not clear [6,7]. In reported families with $I 49 T$ alteration, the altered gene did not happen to segregate with melanoma [8]. Here we report a family with germline CDKN2A I49T alteration and multiple family members affected with cancer. In at least two members, the germline mutation was confirmed. We also show the potential of germline CDKN2A I49T alteration as a targetable driver mutation, as the refractory osteosarcoma went into a prolonged remission after treatment with CDK4/6 inhibitor.
Pathology was reviewed and confirmed at Community Regional Medical Center, Fresno, California after reviewing of all samples with microscopic histology and immune-histo-chemistry (IHC) evaluation for vimentin, pancytokeratin, CD34, S100, desmin, SMA, EMA, CK7, CK20, STAT6, myogenin, ERG, SALL4, Beta-Catenin and MUC4. The pathology samples were additionally reviewed separately at University of California, San Francisco (UCSF) as well as in University of Southern California (USF). Tumor mutational analysis was done by commercially available FoundationOne ${ }^{\mathrm{TM}}$ test (Foundation Medicine, Cambridge, Massachusetts) on the paraffin block of the formaldehyde fixed samples of GIST tumor and osteosarcoma. Germline mutation tests were done on the peripheral blood by commercially available test CancerNext (Ambry Genetics, Aliso Viejo, California). Response to the treatment was monitored by serial computed tomography (CT) imaging.

\section{Case Presentation}

A 28 years old male with no prior significant medical or surgical history presented to ER with progressive shortness of breath, bipedal swelling. His symptoms were worsening over course of a month. Chest $\mathrm{X}$-ray and computed tomograms (CT scans) of the chest showed a large chest mass emanating from the left side rib, causing severe extrinsic compression on the mediastinum and invading through thoracic spinal column. Patient underwent surgical stabilization of thoracic vertebrae, during which, tissue samples were obtained from the hypervascular lesion invading into the vertebral bodies. Positron 
Citation: Tehrani OS, Abdulhaq H, Delozier CD (2019) Potential of Germline CDKN2A I49T as a Targetable Driver Mutation: Prolonged Control of Refractory Osteosarcoma with CDK4/6 Inhibitor in a Familial Cancer. J Carcinog Mutagen 10: 331. doi:10.4172/2157-2518.1000331

Page 2 of 4

emission and computed tomography (PET/CT) obtained after this operation showed a low activity tumor with higher activity in certain areas of the tumor, which was attributed to the biopsied spots. Patient underwent a second surgical operation with sternotomy, left thoracotomy, resection of the chest wall mass with en-bloc tumor resection and partial excision of the left second rib. Surgical and pathology report showed gross residual disease. Post resection, patient recovered well. Pathology confirmed low grade osteosarcoma with spindle shape cells, only positive for vimentin, and negative for pancytokeratin, CD34, S100, desmin, SMA, EMA, CK7, CK20, STAT6, myogenin, ERG, SALL4, Beta-Catenin and MUC4. Final tissue diagnosis was periosteal osteosarcoma of the rib with secondary aneurismal bone cyst-like degeneration and possible focal dedifferentiation into a high-grade osteosarcoma. Post-operation CT scan showed remaining lesions in the mediastinum as well as paraspinal regions. To identify whether these are metastatic foci, biopsy was obtained through endobronchial ultrasound (EBUS). Pathology of these biopsies was compatible with the original tumor histology, confirming metastasis. Based on these findings, case was discussed in multidisciplinary tumor board. Due to the distribution of the residual disease in mediastinum, ribs and spine, and also the low-grade nature of the bulk of the disease, adjuvant radiation was not recommended. Therefore, sample was sent for somatic genetic mutational analysis and in parallel, patient was treated with 4 cycles of systemic chemotherapy with gemcitabine $900 \mathrm{mg} / \mathrm{m}^{2}$ (day 1 and 8) and docetaxel $100 \mathrm{mg} / \mathrm{m}^{2}$ (day 8 ) on a 21-day cycle. Re-imaging after 3 months of chemotherapy showed progressive disease. Case was discussed again in the multidisciplinary tumor board and surgical resection was not recommended. Patient was also not interested in pursuing clinical trials at this time.

Next generation sequencing of osteosarcoma showed gene alterations in the following genes: $C D K N 2 A, T S C 1, C D K 4, K D M 5 A$, PBRM1, KRAS, PAG1, NUP98, NOD1, HNF1A, DTX1, HDAC7. Allele frequency showed that $C D K N 2 A$ mutation has frequency of $47 \%$.

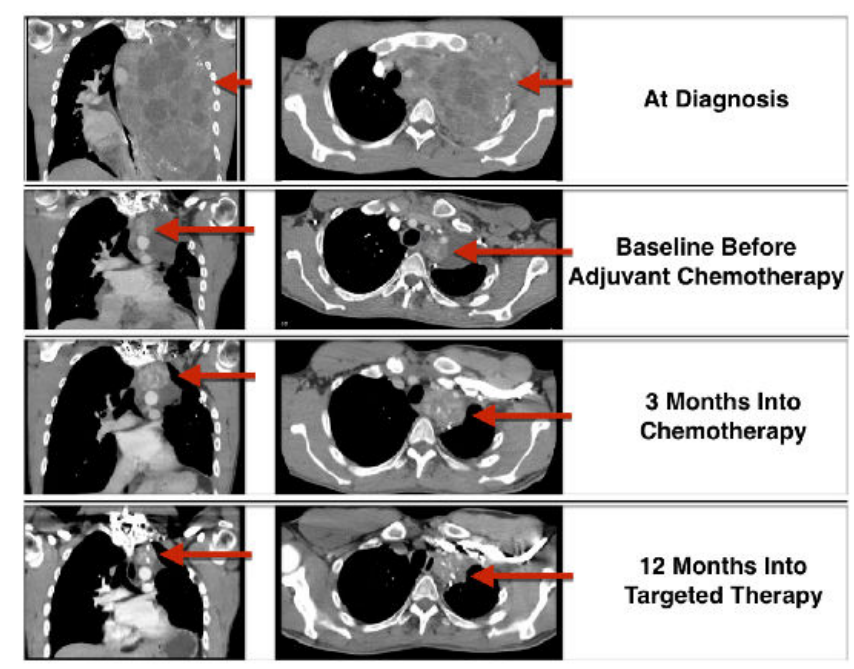

Figure 1: Sagittal and transverse computed tomography images of the osteosarcoma at diagnosis, before chemotherapy, after 3 months of chemotherapy and 12 months into targeted therapy are shown. Arrows point to the tumor.
Due to the progressive disease with CDKN2A alteration, patient was started on CDK4/6 inhibitor palbociclib $125 \mathrm{mg}$ daily for 21 consecutive days and 7 days off treatment in a 28-day cycle. Follow up images after 3 months showed regression of the disease in the mediastinum, and eventually calcification formed in the mediastinal lesion on imaging 12 months on treatment (Figure 1). Patient has tolerated the treatment without complications and with normal blood tests. Absolute neutrophil counts remained within normal limits over the course of the next 12 months. Patient remained asymptomatic and fully functional.

Family history of the patient was positive for patient's mother with gastrointestinal stromal tumor (GIST) of small intestine, diagnosed at age 45. She was treated successfully with surgical resection and adjuvant imatinib. Besides patient's mother, there was significant family history of cancer. Maternal grandmother was also diagnosed with breast cancer at age 35, died at age 40 . Four of grandmother's seven siblings reportedly had cancer; most of them developed cancer prior to the age of 45 . Two of the affected members had lung cancer and one had throat cancer (Figure 2).

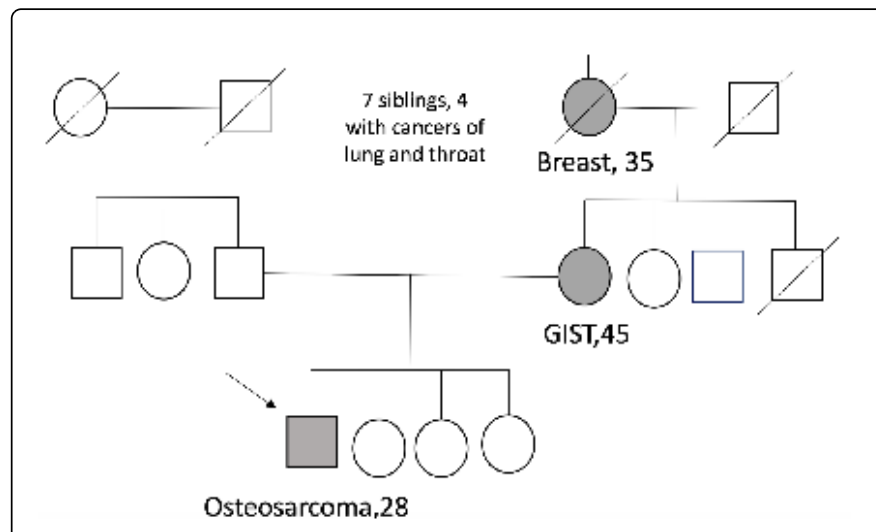

Figure 2: Pedigree of the family with affected members with germline mutation of DCKN2A 149 T alteration. The oblique arrow is pointing at the proband. Age at diagnosis and the type of cancer of the affected family members are shown with full circles and squares.

Notably none of them had history of smoking. Mother's GIST tumor was also analyzed with next generation sequencing and showed mutation in the following genes: CDKN2A, c-KIT, RB1, SPTA1, $E R B B 2, L R P 1 B, E P H A 5, P O L E$. Notably, allele frequency was close to $50 \%$ in several genes: CDKN2A (47\%), RB1 (56\%), SPTA1 (50\%), EPHA5 (48\%), and POLE (52\%) (Table 1). Since tumor samples of patient and his mother showed CDKN2A I49T alteration, patient and his mother's were tested for germline mutation and both were found to be positive for germline CDKN2A I49T mutation.

\begin{tabular}{|l|l|l|l|l|l|}
\hline \multicolumn{3}{|c|}{ Patient } & \multicolumn{3}{c|}{ Mother } \\
\hline Gene & Protein effect & $\begin{array}{c}\text { Allele } \\
\text { Frequency }\end{array}$ & Gene & Protein effect & $\begin{array}{c}\text { Allele } \\
\text { Frequency }\end{array}$ \\
\hline CDKN2A & I49T & $47 \%$ & $\begin{array}{l}\text { CDKN2 } \\
A\end{array}$ & $149 T$ & $47 \%$ \\
\hline TSC1 & D24FS*4 & $7 \%$ & KIT & W557S & $37 \%$ \\
\hline CDK4 & Amplified x7 & & RB1 & L809fs*17 & $56 \%$ \\
\hline
\end{tabular}


Page 3 of 4

\begin{tabular}{|l|l|l|l|l|l|}
\hline KDM5A & Amplified $\mathrm{x} 7$ & & SPTA1 & R891* & $50 \%$ \\
\hline PBRM1 & Y1577D & $51 \%$ & ERBB2 & Q1148L & $7 \%$ \\
\hline KRAS & M189L & $77 \%$ & LRP1B & 1844M & $48 \% \%$ \\
\hline PAG1 & E281K & $50 \%$ & EPHA5 & S124F & $10 \%$ \\
\hline NUP98 & D1757V & $50 \%$ & POLE & G380S & $52 \%$ \\
\hline NOD1 & V268M & $52 \%$ & & & \\
\hline HNF1A & Amplified $x 6$ & & & & \\
\hline DTX1 & Amplified $x 6$ & & & & \\
\hline HDAC7 & Amplified $x 7$ & & & & \\
\hline
\end{tabular}

\section{Conclusion}

Germline CDKN2A I49T mutation is a potentially targetable driver mutation and can be associated with familial cancer.

\section{Declarations}

Ethics approval and consent to participate was waived, obtained by UCSF Fresno institutional review board.

Consent for publication was obtained from the patient.

\section{Availability of Data and Materials}

Please contact author for data requests.

Table 1: Altered genes, type of genetic alteration and allele frequencies reported in the osteosarcoma and gastrointestinal stromal tumor (GIST). Coding alterations are reported with the amino acid alterations or the copy number as amplifications.

\section{Discussion}

Pathogenicity and association of CDKN2A $I 49 T$ with familial cancer has been a matter of controversy. As noticed in FAMMM, CDKN2A I49T did not segregate with melanoma [8]. Additionally, while somatic CDKN2A deletion is frequently reported in human osteosarcoma, germline $C D K N 2 A$ mutation is common in canine osteosarcoma $[1,9]$. In the present study, none of the family members had melanoma, dysplastic nevi, glioblastoma or pancreatic cancer, common features of FMMM. Instead, there was a range of early age breast cancer, lung cancer, head and neck cancer, GIST and osteosarcoma. It is possible that families affected by germline CDKN2A I49T mutation carry a different risk of malignancies compared to those with FAMMM.

Osteosarcoma is usually divided into high grade and low-grade subtypes. Most of the non-metastatic low-grade osteosarcomas are treated by surgical resection. Treating low grade osteosarcoma with islands of high grade disease has been a matter of controversy. In a study in 2015 it was suggested that if high grade is less than $50 \%$ of the tumor, complete surgical resection is the only necessary treatment [10]. The current case had slow growing osteosarcoma with small islands of high grade cancer and despite several attempts, total resection was not possible. Imaging showed that despite combination chemotherapy, tumor was growing and forming new lesions. The main challenge in this case, was to find a targetable driver mutation.

It is known that $C D K N 2 A I 49 T$ alteration affects p16 [6,7]. Cellular and molecular studies in $C D K N 2 A I 49 T$ mutant cells have shown a different intracellular distribution of p16 [11]. Among products of CDKN2A, p16, is a natural inhibitor of CDK4/6 [12]. Therefore in the osteosarcoma case with germline CDKN2A mutation, a CDK4/6 inhibitor could be a potential candidate to replace the dysfunctional protein. Treatment with palbociclib, a CDK4/6 inhibitor, resulted in prolonged control of the disease, supporting the potential of germline mutation in $C D K 4 / 6$ I49T for targeted tumor therapy. Other targetable germline mutations include BRCA1,BRCA2 and $A T M$ in breast and ovarian cancers. Such cancers are candidates for treatment with ADPribose polymerase (PARP) inhibitors [13]. Further studies are needed to explore and confirm the potential of CDK4/6 inhibitors in treating patients with germline $C D K 4 / 6$ mutations.

\section{Competing Interests}

The authors declare that they have no competing interests.

\section{Funding}

No funding support in the design of the study and collection, analysis, and interpretation of data and in writing.

\section{References}

1. Nielsen GP, Burns KL, Rosenberg AE, Louis DN. CDKN2A (1998) Gene deletions and loss of p16 expression occur in osteosarcomas that lack RB alterations. Am J Pathol 153: 159-163.

2. Goldstein AM, Fraser MC, Struewing JP, Hussussian CJ, Ranade K, et al. (1995) Increased risk of pancreatic cancer in melanoma-prone kindreds with p16INK4 mutations. N Engl J Med 333: 970-974.

3. de Snoo FA, Bishop DT, Bergman W, van Leeuwen I, van der Drift C, et al. (2008) Increased risk of cancer other than melanoma in CDKN2A founder mutation (p16-Leiden)-positive melanoma families. Clin Cancer Res Off J Am Assoc Cancer Res 14: 7151-7157.

4. Bahuau M, Vidaud D, Jenkins RB, Bièche I, Kimmel DW, et al. (1998) Germ-line deletion involving the INK4 locus in familial proneness to melanoma and nervous system tumors. Cancer Res 58: 2298-2303.

5. Yarbrough WG, Aprelikova O, Pei H, Olshan AF, Liu ET (1996) Familial tumor syndrome associated with a germline nonfunctional p16INK4a allele. J Natl Cancer Inst 88: 1489-1491.

6. Lin Y-C, Diccianni MB, Kim Y, Lin H-H, Lee C-H, et al. (2007) Human p16gamma, a novel transcriptional variant of p16(INK4A), coexpresses with p16(INK4A) in cancer cells and inhibits cell-cycle progression. Oncogene 26: 7017-7027.

7. Rizos H, Darmanian AP, Mann GJ, Kefford RF (2000) Two arginine rich domains in the p14ARF tumour suppressor mediate nucleolar localization. Oncogene 19: 2978-2985.

8. Hussussian CJ, Struewing JP, Goldstein AM, Higgins PA, Ally DS, et al. (1994) Germline p16 mutations in familial melanoma. Nat Genet 8: 15-21.

9. Karlsson EK, Sigurdsson S, Ivansson E, Thomas R, Elvers I, et al. (2013) Genome-wide analyses implicate 33 loci in heritable dog osteosarcoma, including regulatory variants near CDKN2A/B. Genome Biol 14: R132.

10. Righi A, Paioli A, Dei Tos AP, Gambarotti M, Palmerini E, et al. (2015) High-grade focal areas in low-grade central osteosarcoma: high-grade or still low-grade osteosarcoma? Clin Sarcoma Res 5: 23.

11. Serrano M, Hannon GJ, Beach D (1993) A new regulatory motif in cellcycle control causing specific inhibition of cyclin D/CDK4. Nature 366: 704-707.

12. Walker GJ, Gabrielli BG, Castellano M, Hayward NK (1999) Functional reassessment of P16 variants using a transfection-based assay. Int J Cancer 82: 305-312. 
Citation: Tehrani OS, Abdulhaq H, Delozier CD (2019) Potential of Germline CDKN2A I49T as a Targetable Driver Mutation: Prolonged Control of Refractory Osteosarcoma with CDK4/6 Inhibitor in a Familial Cancer. J Carcinog Mutagen 10: 331. doi:10.4172/2157-2518.1000331

Page 4 of 4

13. Helleday T (2016) PARP inhibitor receives FDA breakthrough therapy designation in castration resistant prostate cancer: beyond germline BRCA mutations. Ann Oncol J Eur Soc Med Oncol 27: 755-757. 